\title{
ANÁLISE DA PRODUÇÃO DE CONHECIMENTO SOBRE O HIV/AIDS EM RESUMOS DE ARTIGOS EM PERIÓDICOS BRASILEIROS DE ENFERMAGEM, NO PERÍODO DE 1980 A 2005 ANALYSIS OF HIV/SIDA KNOWLEDGE PRODUCTION IN ABSTRACTS IN BRAZILIAN NURSING JOURNALS BETWEEN 1980 AND 2005 EL ANÁLISIS DE LA PRODUCCIÓN DEL CONOCIMIENTO SOBRE EL VIH/SIDA EN RESÚMENES DE ARTÍCULOS EN PERIÓDICOS BRASILEÑOS DE ENFERMERÍA EN EL PERÍODO DE 1980 AL 2005
}

\author{
Denize Cristina de Oliveira', Tadeu Lessa da Costa ${ }^{2}$, Antônio Marcos Tosoli Gomes ${ }^{3}$, Sonia Acioli ${ }^{4}$, Gláucia Alexandre \\ Formozo $0^{2}$, Ariádina Heringer ${ }^{2}$, Alain Giami ${ }^{5}$
}

${ }^{1}$ Doutora em Saúde Pública. Professora Titular da Faculdade de Enfermagem da Universidade do Estado do Rio de Janeiro
(FENF/UERJ).
${ }^{2}$ Mestrando(a) do Programa de Pós-Graduação em Enfermagem da FENF/UERJ.
${ }^{3}$ Doutor em Enfermagem. Professor Assistente da FENF/UERJ.
${ }^{4}$ Doutora em Saúde Coletiva. Professora Adjunto do Departamento de Saúde Pública e do Programa de Pós-Graduação da
FENF/UERJ.
${ }^{5}$ Doutor em Psicologia. Diretor de Pesquisa no Institut National de la Santé el de la Recherche Médicale (INSERM), em Le
Kremlin Bicêtre, France. Professor da Faculte de Médecine, na Université Paris-Sud, em Le Kremlin Bicêtre, France.

PALAVRAS-CHAVE: Enfermagem. Saúde pública. Pesquisa em enfermagem. Infecções por HIV.

KEYWORDS: Nursing. Public health. Nursing research. HIV infections.

PALABRAS CLAVE: Enfermería. Salud pública. Investigación en enfermería. Infecciones por VIH.
RESUMO: Trata-se de uma pesquisa quantitativa, descritiva, tendo por objetivos identificar e analisar a literatura científica sobre HIV/AIDS, divulgada em periódicos brasileiros de enfermagem, no período de 1980 a 2005. Pesquisaram-se os bancos de dados: BVS-Enfermagem, com descritores "HIV or AIDS"; LILACS, SCIELO, Portal CAPES e BIREME, com os descritores "HIV or AIDS and enfermagem". Como idiomas, considerou-se português, inglês e espanhol. Constatou-se que a maioria dos estudos sobre HIV/AIDS foi publicada entre 2000 e 2005, tendo maior quantitativo em metodologias qualitativas descritivas. Os clientes soropositivos ao HIV/AIDS foram os sujeitos mais freqüentes, com um destaque para o hospital como campo de estudo. A temática predominante foi o cuidar de clientes com HIV/AIDS. Conclui-se que há interesse pela temática no campo do HIV/AIDS e que esse interesse está associado às práticas profissionais desenvolvidas no campo.

ABSTRACT: This is a quantitative and descriptive study. Its objective is identify and to analyze the scientific literature about HIV/SIDA in Brazilian nursing periodicals, between 1980 and 2005. The databases searched were: BVS-Enfermagem, with descriptors "hiv or aids"; and LILACS, SCIELO, Portal CAPES and BIREME, with descriptors "hiv or aids and nursing". The languages considered in the search were Portuguese, Spanish, and English. The majority of these studies on HIV/SIDA were published between 2000 and 2005, of which a majority were qualitative, descriptive studies. The largest of the groups studied were patients with HIV or SIDA and the used location for data collection used most was the hospital. The predominant theme was nursing care for patients with HIV or SIDA. Further reflection is necessary about the tendencies of nursing research about HIV/SIDA.

RESUMEN: Se trata de una investigación cuantitativa, tipo descriptivo, que tuvo como objetivos identificar y analizar la literatura científica publicada en los periódicos brasileños de enfermería sobre VIH/SIDA, en el período de 1980 al 2005. Los bancos de datos seleccionados fueron: BVS-Enfermería, con descriptores "HIV or AIDS"; LILACS, SCIELO, Portal CAPES y BIREME, con los descriptores "HIV or AIDS and enfermagem". Los idiomas fueron portugués, inglés y español. Los resultados demostraron que la mayoría de los estudios sobre VIH/SIDA fueron publicados en el período de 2000 al 2005, destacándose las investigaciones de abordaje cualitativo descriptivo. Los pacientes con VIH o SIDA seropositivos fueron los sujetos más recientemente. El tema predominante fue cuidar de pacientes con $\mathrm{VIH} / \mathrm{SIDA}$. Se concluye que existe el interés por el VIH/SIDA y que este interés se asocia al conjunto de las prácticas profesionales en el campo de estudio.
Endereço: Denize Cristina de Oliveira

R. General Ribeiro da Costa, 178, Ap. 1.201

22.010-050 - Leme, Rio de Janeiro, RJ.

E-mail: dcouerj@gmail.com
Artigo original: Pesquisa Recebido em: 22 de maio de 2006. Aprovação final: 26 de outubro de 2006. 


\section{INTRODUÇÃO}

Este trabalho vincula-se ao projeto de pesquisa "Representações sociais da AIDS e memória profissional das enfermeiras no Brasil: estudo bicêntrico Rio de Janeiro/Florianópolis", desenvolvido através de uma parceria entre Brasil e França, respectivamente, por meio do Programa de Pós-Graduação da Faculdade de Enfermagem da Universidade do Estado do Rio de Janeiro (FENF/UERJ), do Departamento de Enfermagem da Universidade Federal de Santa Catarina (UFSC) e do Institut National de la Santé et de la Recherche Medicale (INSERM).

Tal projeto, em linhas gerais, visa analisar a memória e as representações sociais acerca da Síndrome da Imunodeficiência Adquirida (AIDS) em grupos de enfermeiros, ao longo da história da epidemia no Brasil, bem como recuperar a trajetória pessoal e profissional de enfermeiros que atuaram ou atuam no cuidado aos sujeitos acometidos por este agravo nas instituições de saúde do Rio de Janeiro e Santa Catarina.

A temática do Vírus da Imunodeficiência Humana (HIV) e da AIDS consiste em um dos grandes problemas contemporâneos da Saúde Pública, trazendo implicações para a sociedade em suas diversas dimensões, perpassando por aspectos objetivos e subjetivos. Deve ser ressaltado que se convive com duas epidemias distintas, embora relacionadas. A primeira refere-se à do HIV, a qual, apesar de possuir maior magnitude, caracteriza-se pela sua considerável invisibilidade. A segunda é a da AIDS, freqüentemente descrita pela sua magnitude estimada em termos de impacto social.

No Brasil, até junho de 2005, foram notificados, aproximadamente, 372 mil casos, dos quais se contabilizou, até dezembro de 2004, 172 mil óbitos. ${ }^{1}$ O impacto do surgimento desta epidemia, no início da década de 80 , foi tal que alguns autores a incluem na categoria daquelas caracterizadas como "pestes", demandando amplas e imediatas respostas do Estado, com uma intensa capacidade para a geração de representações entre os grupos sociais.

No decorrer deste percurso de 25 anos (19802005), estudos acerca do HIV e da AIDS têm sido, então, desenvolvidos e divulgados por diversas áreas do conhecimento, entre as quais, a enfermagem. Nesse campo a epidemia repercute em diferentes aspectos do seu processo de trabalho, e também, do cuidado prestado às pessoas, consistindo, portanto, em uma temática de significativo interesse para esta profissão. ${ }^{2}$
A relevância deste estudo coloca-se no plano do resgate da trajetória seguida pelo conhecimento científico da enfermagem no campo do HIV/AIDS, revelando as modulações sofridas pelo conhecimento acumulado e pela própria área de conhecimentos e práticas. Essa dinâmica, bastante evidente nos estudos bibliográficos, permite repensar a propriedade dos objetos e das orientações teóricas que estão sendo privilegiadas.

Delimita-se, portanto, como objeto para o presente estudo o perfil da literatura científica sobre o HIV/AIDS divulgada em periódicos brasileiros de enfermagem, no período de 1980 a 2005. Como objetivos para esta investigação definiu-se identificar e analisar a literatura científica sobre o HIV/AIDS divulgada em periódicos brasileiros de enfermagem, no período de 1980 a 2005.

\section{TRAJETÓRIA METODOLÓGICA}

Trata-se de um estudo descritivo, pautado na abordagem quantitativa. A busca pelos artigos divulgados em periódicos brasileiros de enfermagem sobre o HIV/AIDS foi efetuada nas bases de dados: "Biblioteca Virtual em Saúde - Enfermagem" (BVS - Enfermagem); "Literatura Latino-Americana e do Caribe em Ciências da Saúde" (LILACS); “ "Scientific Eletronic Library Online" (SciELO). Na primeira, utilizaram-se como descritores "hiv or aids", uma vez que consiste numa base específica de enfermagem e, nas demais, "hiv or aids and enfermagem".

Neste processo, tendo em vista os objetivos do estudo, foram excluídos os artigos publicados em periódicos que não eram específicos de enfermagem ou não editados no Brasil.

Para abarcar o maior número possível de artigos, realizou-se, ainda, busca por periódicos brasileiros de enfermagem na Biblioteca Regional de Medicina (BIREME) e no Portal da Coordenação de Aperfeiçoamento de Pessoal de Nível Superior (CAPES), por meio de seus respectivos sítios eletrônicos, busca essa baseada na classificação "Qualis das Áreas", disponibilizada no Portal CAPES, referente à área de enfermagem e ao ano de 2004. Os descritores aplicados, igualmente, foram "hiv or aids", considerando já serem publicações de enfermagem.

As buscas ocorreram durante os meses de fevereiro a abril de 2006, tendo sido incluídos os idiomas português, inglês e espanhol, com o recorte temporal referente ao período de janeiro de 1980 a dezembro de 2005. 
Foram identificados 26 periódicos brasileiros de enfermagem, entre os quais 15 contavam com artigos indexados sobre a temática. Obteve-se, assim, um total de 152 artigos, dos quais $34(22,4 \%)$ apresentavam texto completo e resumo, $116(76,3 \%)$ somente resumo e $2(1,3 \%)$ não possuíam resumo ou texto completo. Assim, optou-se por trabalhar com resumos e com o último tipo de registro citado $(\mathrm{n}=152)$.

Foram adotadas as seguintes variáveis de caracterização das publicações para análise: o período de publicação; os tipos de estudo; periódicos em que foram publicados; regiões e estados de publicação e coleta de dados; circulação, segundo a classificação "Qualis das Áreas" concernente ao ano de 2004; idiomas; tipos de estudo no que tange à metodologia; referenciais teóricos utilizados; sujeitos das pesquisas; campos de estudo ou coleta de dados; e temáticas abordadas.

Em relação às variáveis "tipos de estudo/metodologia" e "temáticas abordadas", foi necessário a aplicação de análise de conteúdo temática, seguindo procedimentos de categorização definidos por Bardin. ${ }^{3}$ A justificativa para a escolha dessa técnica é dada pela dispersão e não menção clara e objetiva do conteúdo das variáveis referidas, nos estudos analisados, ou seja, os resumos, em sua maioria, não mencionavam o tipo de estudo, e as temáticas eram numerosas.

Os resultados foram sistematizados e apresentados por meio de tabelas e gráficos, tendo sido a sua discussão pautada na literatura científica de enfermagem ou de outras áreas do conhecimento que se mostraram pertinentes à compreensão dos achados.

\section{RESULTADOS}

O primeiro artigo sobre a epidemia do HIV/ AIDS foi publicado em 1986, na Revista Paulista de Enfermagem, tratando-se de uma revisão de literatura sobre a temática. Tal publicação pode ser considerada uma resposta pronta da enfermagem à emergência do HIV/AIDS no Brasil, tendo em vista que o primeiro registro de caso de AIDS no país, se deu em 1982, em São Paulo; em 1983, uma equipe de profissionais de saúde inaugurou no Instituto de Saúde da Secretaria de Saúde do estado de São Paulo o que viria, posteriormente, a ser o primeiro Programa de Atenção e Prevenção de AIDS no país; a identificação do agente etiológico ocorreu em 1983, na França e nos Estados Unidos, com ampla divulgação na mídia; e, ainda, o primeiro teste sorológico, o Ensaio Imunoenzimático Ligado à Enzima (ELISA), surgiu, apenas, no final do ano de $1984 .^{4}$
Além disso, é interessante ressaltar que, de acordo com o primeiro Boletim Epidemiológico de AIDS, editado em 1987, entre 1982 e 1987 somavam-se 1.906 casos no país e a edição de 1996, na qual observa-se a consolidação dos dados até então, aponta para 4.287 o número de casos no mesmo período, ainda que, em parte, retrospectivos. ${ }^{1}$

A partir desta data, são encontrados artigos em todos os anos do período delimitado para esta pesquisa, o que demonstra o interesse da área de enfermagem por temas da atualidade, já que se observou a criação de um programa de AIDS de abrangência nacional, em 1986, a partir da experiência de São Paulo, passando este agravo a possuir notificação compulsória. ${ }^{4}$ Desde então, como pode ser observado na Tabela 1 , o número de publicações mostrou-se crescente, sendo o período com maior quantitativo o de 2000 a 2005.

Este crescimento do número de publicações parece refletir a inexistência de cura e a cronificação do agravo em questão, além do advento e da distribuição universal e gratuita da terapia anti-retroviral, a partir de 1996 pelo Ministério da Saúde. ${ }^{5}$ Este cenário despertou o interesse de diversos pesquisadores para a epidemia, trazendo novas questões como a gestão de recursos financeiros e insumos variados, os desafios de manipulação de tecnologias pelas famílias e pela unidade de saúde, e, especialmente, a implementação de uma política e de um programa nacional de DST/AIDS. Ressaltam-se, ainda, as alterações ocorridas na trajetória epidemiológica da síndrome, as quais geraram novas demandas e motivaram autores que se interessavam pelas áreas da saúde da mulher, da criança e pelo tema família, entre outros. ${ }^{6}$

Tabela 1 - Distribuição dos artigos por período de publicação. Rio de Janeiro, 2006.

\begin{tabular}{ccc}
\hline Período & $\mathrm{f}$ & $\%$ \\
\hline $1980-1989$ & 9 & 5,9 \\
$1990-1999$ & 66 & 43,4 \\
$2000-2005$ & 77 & 50,7 \\
\hline Total & $\mathbf{1 5 2}$ & $\mathbf{1 0 0}$ \\
\hline
\end{tabular}

Quanto ao tipo de estudo, pôde-se constatar um predomínio das pesquisas empíricas, correspondendo a $68 \%$ do total, seguida das análises teóricas (13\%), dados esses semelhantes aos encontrados em 
uma pesquisa bibliográfica acerca da investigação científica em enfermagem no Brasil. ${ }^{10}$ Este resultado parece refletir o esforço que a profissão tem feito para consolidar-se como área de conhecimento, bem como para conhecer as dimensões das inserções concretas da epidemia do HIV/AIDS na sociedade. ${ }^{7}$

O periódico brasileiro de enfermagem com maior quantitativo de publicações acerca da epidemia do HIV/AIDS foi a Revista Latino-Americana de Enfermagem (21,7\%), seguida pela Revista Brasileira de Enfermagem (15,8\%), Revista de Enfermagem UERJ (11,2\%), Revista da Escola de Enfermagem da USP e Revista Gaúcha de Enfermagem $(9,2 \%)$, tal qual disposto na Tabela 2.

Neste ponto é importante ressaltar que o primeiro e o segundo periódicos citados apresentam, de longa data, periodicidade menor, com maior número de artigos publicados por ano, fato concorrente para o resultado do quantitativo de artigos. Além disso, a maior concentração de Escolas de Enfermagem e de Programas de Pós-Graduação na Região Sudeste, especialmente no estado de São Paulo, pode, também, ter contribuído para uma maior participação da Revista Latino-Americana de Enfermagem nas publicações. A Revista Brasileira de Enfermagem, por sua vez, justifica a sua posição por caracterizar-se como o mais antigo e tradicional veículo de divulgação científica da Enfermagem brasileira, refletindo as tendências da área.

Tabela 2 - Distribuição dos artigos segundo os periódicos. Rio de Janeiro, 2006.

\begin{tabular}{lcc}
\hline \multicolumn{1}{c}{ Periódicos } & f & \% \\
\hline Revista Latino-Americana de Enfermagem & 33 & 21,7 \\
Revista Brasileira de Enfermagem & 24 & 15,8 \\
Revista de Enfermagem da UERJ & 17 & 11,2 \\
Revista da Escola de Enfermagem da USP & 16 & 10,5 \\
Revista Gaúcha de Enfermagem & 14 & 9,2 \\
Revista Paulista de Enfermagem & 10 & 6,6 \\
Escola Anna Nery Revista de Enfermagem & 8 & 5,3 \\
Revista Mineira de Enfermagem & 6 & 3,9 \\
Acta Paulista de Enfermagem & 5 & 3,3 \\
Outros & 19 & 12,5 \\
\hline Total & $\mathbf{1 5 2}$ & $\mathbf{1 0 0}$ \\
\hline
\end{tabular}

Em relação à distribuição dos artigos entre os periódicos editados por regiões do país, constatou-se que 104 (68,4\%) encontravam-se na região Sudeste, no contexto da qual destacaram-se os estados de São Paulo, com 70, correspondente à $46 \%$ do total, e Rio de Janeiro, com 28, equivalente à $18,4 \%$ do total. A região Centro-Oeste, mais especificamente o Distrito Federal, apresentou 24 (15,8\%) artigos publicados, concernentes à Revista Brasileira de Enfermagem, já discutida, anteriormente. Na região Sul, identificou-se o quantitativo de $22(14,5 \%)$ artigos, sendo o Rio Grande do Sul o estado com maior número, 14 (9,2\%) artigos. O Nordeste contou somente com $2(1,3 \%)$ artigos publicados em um periódico editado da região e, no Norte não se evidenciou a edição de periódicos de enfermagem com artigos acerca da epidemia do HIV/AIDS.

Somado a isto, não obstante 63 (41,4\%) resumos dos artigos não terem informado a localidade de desenvolvimento das pesquisas empíricas, entre aqueles em que tal informação existia (51), observou-se, também, predominância do estado de São Paulo, com 20 (13,1\%) resumos, seguido pelo Rio de Janeiro, com 12 (7,9\%), Santa Catarina, com 5 (3,3\%) e Rio Grande do Sul, com 4 (2,6\%). Faz-se mister salientar que 38 (25\%) do total dos 152 resumos, por serem relatos de experiência, análises teóricas ou pesquisas bibliográficas, não apresentaram essa informação.

Os resultados acima reforçam a análise $\mathrm{da}$ variável anterior, e se relacionam, dentre outros elementos, ao fato de a região Sudeste ter sido a primeira a implantar e, depois, a concentrar Programas de Pós-Graduação em Enfermagem, especialmente nos estados de São Paulo e Rio de Janeiro, onde também foram notificados os casos iniciais de HIV/AIDS, e, ainda hoje, registra maior prevalência e a segunda maior incidência do país, com o último estado mencionado respondendo pelo maior número de casos no ano de 2004. ${ }^{1}$ É interessante notar que, não obstante a incipiente publicação referente às regiões Nordeste e Norte, estas apresentam taxa de incidência de casos de HIV/AIDS em ascensão. ${ }^{1}$ Mesmo levando-se em consideração que os pesquisadores do Nordeste tendem a publicar em periódicos da região Sudeste, ou que ainda realizem suas pesquisas nesta região do país em função da concentração dos cursos de pós-graduação, observa-se a existência de uma décalage entre o perfil epidemiológico da epidemia naquela região e a explicitação das preocupações dos pesquisadores. 
Considerando a avaliação "Qualis das áreas" disponibilizada pela CAPES, correspondente ao ano de 2004, 134 (88\%) dos artigos encontravam-se em periódicos com circulação internacional, sendo 149 (98\%) publicados em língua portuguesa, o que propicia maior consulta e incorporação dos conhecimentos no Brasil. Em contrapartida, a língua portuguesa tende a dificultar a apropriação do conhecimento produzido no Brasil em outros países, haja vista o número reduzido de regiões do mundo nas quais este é o idioma oficial ou tem presença marcante.

No que tange à metodologia, a maior parte dos trabalhos foram qualitativos descritivos, correspondendo a $88(57,9 \%)$ resumos. Esse tipo de pesquisa tem como foco a descrição dos fenômenos escolhidos para estudo, baseados na observação, explicitação e classificação da realidade de interesse, podendo ser dividida em pesquisa bibliográfica, na qual se procede à consulta da literatura, seleção e registro de tópicos pertinentes; e pesquisa de campo, a qual se debruça sobre os fenômenos em cenários naturais, com profundo escrutínio das práticas, comportamentos e atitudes das pessoas ou grupos. ${ }^{8}$

Por sua vez, a abordagem qualitativa se ocupa do aprofundamento e da compreensão da subjetividade humana, seja ela coletiva ou social, bem como da compreensão dos posicionamentos diante dos objetos estudados. Esta questão vem sendo objeto de preocupação dos pesquisadores de enfermagem, desde a realização do $1^{\circ}$ Seminário Nacional de Pesquisa em Enfermagem (SENPE), em 1979, tendo sido, em 1988, promovido o $1^{\circ}$ Encontro Interamericano de Pesquisa Qualitativa em Enfermagem, com o intuito de difundir esta metodologia.

Em um estudo sobre a produção de conhecimento em enfermagem em nível de doutorado, constatou-se, igualmente, uma predominância e uma clara tendência para a utilização da abordagem qualitativa pelos autores das teses. ${ }^{9}$ Neste sentido, pode-se dizer que o afastamento da enfermagem do modelo biomédico, observado nas duas últimas décadas, associado à inclinação deste campo para as ciências humanas e sociais, aponta para a necessidade de especificação da subjetividade implicada na saúde. Além disso, permite conceber a relação coletivo-individual como uma relação dialética, na qual a individualidade não pode ser pensada sem a sua contrapartida social, e ainda possibilita o reconhecimento de uma forma de pensamento natural, com o mesmo status social do conhecimento científico, caracterizada pelo conhecimento do senso comum. ${ }^{10}$

Tratando-se especificamente do HIV/AIDS, tal abordagem mostra-se útil para a enfermagem, uma vez que este campo tem como foco as pessoas, para as quais o referido agravo à saúde traz importantes implicações, ${ }^{6}$ bem como para a própria prática profissional, em suas dimensões objetivas e subjetivas., ${ }^{2,11}$

Quanto aos referenciais teóricos adotados nos estudos, observou-se que, na maior parte $(63,8 \%)$, os mesmos não se encontravam explícitos. Dentre os resumos que apresentavam essa informação (55), 14 (9,2\%) foram desenvolvidos à luz de Teorias de Enfermagem e 7 (4,6\%) da Teoria das Representações Sociais.

As teorias de enfermagem são aportes epistemológicos relevantes para a prática profissional e para o campo de saber, pois orientam as práticas, auxiliando a equipe de enfermagem na identificação das necessidades dos clientes, favorecem a capacidade de descrever e explicar da realidade da assistência, propiciando, assim, subsídios para a intervenção. ${ }^{12}$ Essas características das teorias podem ter sido responsáveis por sua utilização como referenciais para estas pesquisas.

A Teoria das Representações Sociais, em sua orientação psicossocial, tem sido cada vez mais utilizada na área de saúde. Isto, especialmente, nos estudos nos quais importe ter acesso ao conhecimento social que orienta as práticas de uma dada população quanto aos problemas de saúde. ${ }^{10}$

Em um estudo no qual se analisou a utilização da Teoria das Representações Sociais no Brasil, no período de 1988 a 1997, constatou-se que a área da saúde contribuiu com $31,7 \%$ dos trabalhos, no interior da qual a enfermagem apresentou maior quantitativo, representado por $48,1 \%$ dos trabalhos, indicando ter esta Teoria pertinência para a abordagem das problemáticas de interesse para os pesquisadores da área. ${ }^{10}$

Em relação aos sujeitos escolhidos para as pesquisas empíricas, como pode ser observado na Tabela 3, houve maior interesse pela categoria de clientes soropositivos ao HIV ou com AIDS, com 13,1\% dos estudos; seguida pela de mulheres, com 9,9\%; adolescentes, escolares e crianças, com $8,6 \%$; equipe de enfermagem, com $7,9 \%$; e enfermeiros, com $6,6 \%$. 
Tabela 3 - Distribuição dos artigos conforme a delimitação dos sujeitos das pesquisas empíricas. Rio de Janeiro, 2006.

\begin{tabular}{lcc}
\hline \multicolumn{1}{c}{ Categoria de sujeitos } & $\mathbf{f}$ & $\mathbf{\%}$ \\
\hline Clientes soropositivos ao HIV ou com AIDS & 20 & 13,1 \\
Mulheres & 15 & 9,9 \\
Adolescentes, escolares e crianças & 13 & 8,6 \\
Equipe de enfermagem & 12 & 7,9 \\
Enfermeiros & 10 & 6,6 \\
Estudantes de enfermagem ou outras áreas & 8 & 5,3 \\
Outros & 15 & 9,9 \\
Não informado & 10 & 6,6 \\
Não se aplica* & 49 & 32,2 \\
\hline Total & $\mathbf{1 5 2}$ & $\mathbf{1 0 0}$ \\
\hline
\end{tabular}

* Refere-se à análise teórica, pesquisa bibliográfica, relatos de experiência e pesquisas documentais.

Todavia, se somados os estudos realizados com enfermeiros e equipe de enfermagem, constata-se que os sujeitos de maior interesse são os profissionais de enfermagem, padrão semelhante ao encontrado em uma tese que analisou pesquisas de enfermagem em nível de doutorado, defendidas em Programas de Pós-Graduação em Enfermagem da Universidade de São Paulo (USP), Escola de Enfermagem de Ribeirão Preto (EERP) e Universidade Federal do Rio de Janeiro (UFRJ), no período de 1992 a $1997 .{ }^{9}$

Este achado corrobora os apontamentos de autores, ${ }^{2,8,11}$ que discutem as implicações trazidas pela epidemia para a prática profissional, em seus múltiplos aspectos, sustentando o surgimento de mudanças significativas nessas práticas, desde as relações com os clientes até a necessidade de ter acesso ao conhecimento produzido acerca do HIV/AIDS.

A delimitação de clientes soropositivos ao HIV para as pesquisas, fundamentalmente, teve como interesse a busca pela compreensão das mudanças ocorridas em suas vidas, em nível biológico, social ou psicológico, bem como das necessidades daí resultantes. Esta seleção traz, também, à baila uma questão dialética, pois ao mesmo tempo em que a indiferenciação dos sujeitos contribui para minorar os preconceitos e a discriminação, descaracteriza os seus traços sociais e históricos. Constata-se, portanto, a necessidade de um aprofundamento nas reflexões sobre este aspecto do delineamento das pesquisas de enfermagem sobre HIV/AIDS, tendo em vista suas implicações para a produção de conhecimentos.
A categoria de mulheres é composta por variados tipos de sujeitos: mulheres, de um modo geral e soropositivas; gestantes, soropositivas ou não; puérperas; moradoras de favela; e cônjuges, soropositivas ou não. $\mathrm{O}$ interesse por tal categoria deveu-se, possivelmente, à feminilização da epidemia do HIV/AIDS, trazendo repercussões em múltiplas dimensões, perpassando questões relacionadas à cotidianidade da vida, aquelas de gênero, e até o aumento da incidência da transmissão vertical do HIV. ${ }^{6}$

A categoria composta por adolescentes, escolares e crianças abarca menores variações, sendo mais freqüentes entre escolares (escolaridade fundamental e médio) e estudos com adolescentes e crianças com soropositividade ao HIV. Esta categoria tem se tornado importante foco de estudo nas últimas décadas, o que se deve a diversos aspectos: ao aumento da transmissão vertical, ao quantitativo expressivo desse grupo na população brasileira e mundial, à elevação do consumo de drogas entre jovens, a maior liberdade sexual com a Revolução Sexual iniciada na década de 50 e o movimento feminista. ${ }^{13,14}$

Ressalte-se a ausência de estudos, desde o início da epidemia, que tivessem como sujeitos explícitos usuários de drogas ou homossexuais, o que pode refletir uma dificuldade vislumbrada na enfermagem para enfrentar situações (e sujeitos) de marginalização social, como observado em estudo realizado com enfermeiras, na França. ${ }^{2}$

No que se refere à variável "campo de estudo" (Tabela 4), identificou-se uma predominância do cenário hospitalar, com 32 (21,1\%) resumos. $\mathrm{Na}$ segunda e terceira maior expressão tem-se as unidades básicas de saúde e ambulatórios, e as instituições de ensino, somando, respectivamente, 13 $(8,6 \%)$ e $12(7,9 \%)$. Por outro lado, se agrupados todos os campos extra-hospitalares, estes passam a ter maior quantitativo $(24,3 \%)$, o que reflete um maior interesse de pesquisadores desse lócus de atenção e o privilegiamento da abordagem preventiva ${ }^{7}$ especialmente em instituições de ensino, porém a presença marcante do hospital indica a necessidade de investimento em outros cenários de pesquisa.

Destaca-se, também, por sua incipiente expressão, os estudos desenvolvidos nos espaços do Terceiro Setor, sobretudo, as Organizações NãoGovernamentais (ONG), que desempenharam significativo papel na mudança das respostas do Estado à epidemia do HIV/AIDS, bem como na alteração de sua própria trajetória. ${ }^{4}$ 
Tabela 4 - Distribuição dos resumos segundo os campos de estudo de pesquisas empíricas. Rio de Janeiro, 2006.

\begin{tabular}{lcc}
\hline \multicolumn{1}{c}{ Campos de estudo } & F & \% \\
\hline Hospitais & 32 & 21,1 \\
Unidades básicas de saúde ou ambulatórios & 13 & 8,6 \\
Instituições de ensino & 12 & 7,9 \\
Regiões, localidades ou favelas & 6 & 3,9 \\
Domicílio & 4 & 2,6 \\
Espaços do terceiro setor & 2 & 1,3 \\
Outros & 11 & 7,2 \\
Não informado & 34 & 22,4 \\
Não se aplica* & 38 & 25 \\
\hline Total & $\mathbf{1 5 2}$ & $\mathbf{1 0 0}$ \\
\hline
\end{tabular}

* Refere-se à análise teórica, pesquisa bibliográfica e relatos de experiência.

Conforme mostra a Figura 1, as principais temáticas abordadas nos resumos analisados foram as seguintes: cuidar de clientes com HIV ou com AIDS (15,1\%); mulher, relações de gênero, gestação e maternidade $(14,5 \%)$; estratégias educativas e preventivas (11,8\%); saúde do adolescente e do escolar (9,9\%); risco de contaminação ocupacional pelo HIV (7,2\%); a (con)vivência com o HIV/AIDS na família, domicílio e intracasais $(7,2 \%)$; representações sociais e práticas em relação ao HIV/AIDS (6,6\%); e dimensões do viver com o HIV/AIDS.
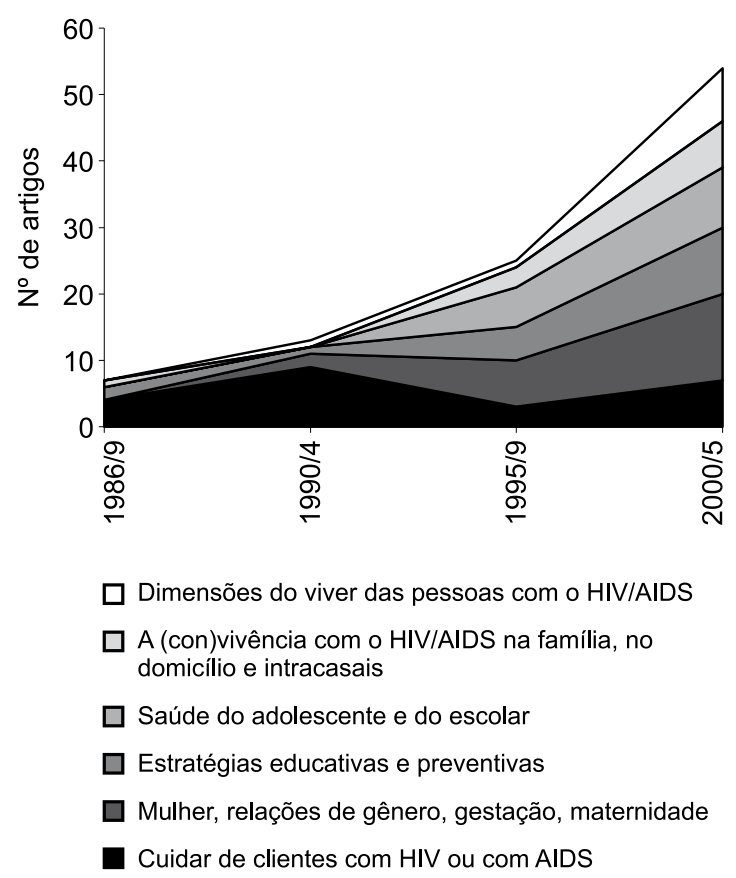

Figura 1 - Distribuição e evolução das principais temáticas por período. Rio de Janeiro, 2006.
É interessante destacar a evolução da abordagem das temáticas, ao longo do período delimitado para este estudo, conforme pode ser observado na figura 1. Percebe-se que, entre 1986 e 1989, há um aumento no número de trabalhos referentes ao cuidado com clientes com AIDS (doentes), com acentuada diminuição na primeira metade da década de 90. Essa diminuição parece estar associada à distribuição gratuita dos anti-retrovirais pelo Ministério da Saúde, aumentando o tempo entre a infecção e o desenvolvimento da AIDS. ${ }^{5} \mathrm{~A}$ partir dessa década, os estudos tendem a focalizar, principalmente, o cuidado às pessoas soropositivas ao HIV, em lugar dos doentes.

Esta mudança na tendência dos estudos publicados, a partir da metade da década de 90, pode ser vislumbrada, também, nas temáticas a (con)vivência com o HIV/AIDS na família, domicílio e intracasais e as dimensões do viver com o HIV/AIDS. Isto se deve ao fato de que os anti-retrovirais vêm proporcionando aumento no tempo e na esperança de vida, porém, seu alto custo e efeitos colaterais, associados à inexistência de cura para o agravo em questão, têm fomentado investigações sobre a qualidade de vida desses sujeitos. Os últimos trabalhos desenvolvidos com essa clientela avaliam não só a dimensão física implicada, mas também, os aspectos psicossociais. ${ }^{5}$

Outro fato que se observa é o aumento de estudos, sobretudo, a partir do início da década de 90, referentes à temática mulher, relações de gênero, gestação, maternidade e sua relação com a epidemia do HIV/AIDS. Esta tendência pode ser explicada a partir da feminilização da epidemia no Brasil, onde foi mais rápida do que em qualquer outra parte do mundo, trazendo profundas implicações para a vida das mulheres, nas diversas fases de seu ciclo vital. Para elas, pode-se dizer que a epidemia representa uma tripla ameaça: a susceptibilidade à infecção; a possibilidade da transmissão ao bebê; e, quando familiares adoecem pelo agravo, geralmente, cabe-lhes a responsabilidade pelos cuidados em casa. ${ }^{6}$

Um último aspecto que merece destaque é a tendência de incremento de trabalhos relacionados à educação e prevenção da infecção pelo vírus do HIV e à saúde do adolescente e do escolar, os quais apresentam, majoritariamente, caráter preventivo. Como anteriormente, descrito, o interesse pela adolescência vem aumentando nas últimas décadas, entre outras coisas, devido à epidemia do HIV/AIDS que atinge de modo significativo as pessoas nesta fase 
da vida. ${ }^{1,13-14}$ Para ilustrar a magnitude do problema, até o primeiro semestre de 2005 , na faixa etária de 13 à 24 anos, foram notificados 43.598 casos. $^{1}$

Por sua vez, atividades educativas e preventivas sempre foram marcantes ao longo da trajetória histórica da enfermagem brasileira e, no caso do interesse constatado em relação à epidemia em foco, pode estar ligado a incentivos governamentais à prevenção da disseminação do $\mathrm{HIV}^{7}$ e ao fato de não se vislumbrar a sua cura, sendo a prevenção a principal forma do seu enfrentamento. ${ }^{15}$

\section{CONSIDERAÇÕES FINAIS}

No decorrer desta pesquisa, que analisou a produção de conhecimentos acerca da epidemia do HIV/AIDS, divulgada em periódicos brasileiros de enfermagem, realizada através dos resumos dos artigos, constatou-se, como principais resultados, que a maior parte dos trabalhos concentrou-se entre 2000 e 2005, tendo sido o tipo de estudo mais significativo a pesquisa empírica. Quanto à temporalidade dos trabalhos, observou-se também a precocidade da primeira publicação na enfermagem.

A região que mais apresentou periódicos abordando a temática foi a Sudeste, bem como na qual foram desenvolvidas o maior número de pesquisas empíricas, com destaque para os estados de São Paulo e Rio de Janeiro. Os periódicos com maior quantitativo de artigos foram a Revista Latino-Americana de Enfermagem, Revista Brasileira de Enfermagem e Revista de Enfermagem UERJ.

Em relação à metodologia, houve a predominância de estudos qualitativos descritivos, sendo os sujeitos da maior parcela dos estudos, clientes soropositivos ao HIV. Destacaram-se também, quantitativamente, mulheres, com variadas inserções sociais, adolescentes, escolares e crianças, equipe de enfermagem e enfermeiros.

Quando analisado isoladamente, o campo de estudo mais utilizado foi o hospital, apontando para a valorização de um modelo individualizado de atenção à saúde, no entanto na análise em conjunto, os espaços não hospitalares se destacam.

As temáticas que se sobressaíram foram: o cuidar de clientes soropositivos ao HIV ou com AIDS; mulher, relações de gênero, gestação, maternidade e HIV/AIDS; estratégias educativas e preventivas em relação à epidemia do HIV/AIDS; e a saúde do adolescente e do escolar, as quais evoluíram conforme a dinâmica da epidemia e das respostas governamentais e não-governamentais, no Brasil.

É importante ressaltar a ausência de pesquisas sobre a questão do uso de drogas e homossexualidade relacionadas à epidemia do HIV/AIDS, sobretudo, tendo como sujeitos usuários de drogas e homossexuais, o que pode refletir uma dificuldade do campo da enfermagem de se posicionar frente a tais assuntos, não obstante a relevância da trajetória desse agravo à saúde no cenário nacional.

Pode-se afirmar que a pesquisa em enfermagem é importante pela sua potencialidade de legitimar o fazer, buscar novas maneiras de cuidado, aproximando as dimensões teóricas e práticas da profissão e contribuindo para a melhoria da qualidade de vida da população, ao mesmo tempo em que dá sustentação à prática profissional. ${ }^{10}$

Assim, não obstante a difícil tarefa de analisar uma produção científica tão complexa e variada como a da enfermagem por meio de resumos de artigos, com as limitações daí decorrentes, este estudo pretende contribuir para o entendimento do caminho percorrido pela enfermagem na construção do conhecimento acerca da epidemia do HIV/AIDS.

\section{REFERÊNCIAS}

1 Ministério da Saúde (BR), Secretaria de Vigilância à Saúde. Programa Nacional de Controle e Prevenção de DST/AIDS. Semanas epidemiológicas: $1^{\mathrm{a}}-26^{\mathrm{a}}$ de 2005. Bol. epidemiológico: AIDS DST. 2005 jan-jun; 2 (1): 3-34.

2 Giami A. Enfermeiras frente à AIDS: representações e condutas, permanência e mudanças. Canoas (RS): ULBRA; 1997.

3 Bardin L. Análise de conteúdo. Paris (FR): Edições 70; 1977.

4 Pinel AC. O que é AIDS: 2a visão. São Paulo (SP): Brasiliense; 1996.

5 Gir E, Vaichulonis CG, Oliveira MD. Adesão à terapêutica anti-retroviral por indivíduos com HIV/AIDS assistidos em uma instituição do interior paulista. Rev. Latino-American. Enferm. 2005 SetOut; 13 (5): 634-41.

6 Reis AL, Xavier IM. Mulher e AIDS: rompendo o silêncio de adesão. Rev. Bras. Enferm. 2003 Jan-Fev; 56 (1): 28-34.

7 Rodrigues RM, Bagnato MHS. Pesquisa em enfermagem no Brasil: problematizando a produção de conhecimentos. Rev. Bras. Enferm. 2003 NovDez; 56 (6): 646-50. 
8 Marziale MHP, Rodrigues CM. A produção científica sobre os acidentes de trabalho com material perfurocortante entre trabalhadores de enfermagem. Rev. Latino-American. Enferm. 2002 Jul-Ago; 10 (4): 571-7.

9 Santiago MMA. O saber acadêmico de enfermagem: constituição e representações em três programas de pós-graduação [tese]. Rio de Janeiro (RJ): UFRJ/ Programa de Pós-Graduação em Enfermagem; 2000.

10 Oliveira DC. A enfermagem e as necessidades humanas básicas: o saber/fazer a partir das representações sociais [tese]. Rio de Janeiro (RJ): UERJ/Faculdade de Enfermagem; 2001.

11 Gir E, Silva AM, Costa FPP, Hayashida M. Alterações na prática profissional de enfermeiros de um hospital de ensino do interior paulista, em conseqüência ao surgimento do HIV/AIDS. Rev. Gaúch. Enferm. 2000 Jul; 21 (2): 37-54.

12 George JB. Teorias de enfermagem: fundamentos para a prática profissional. 4a ed. Porto Alegre (RS): Artes Médicas; 2000.

13 Cano MAT, Ferriani MGC, Alves AC, Nakata CY. A produção de conhecimento sobre adolescência na enfermagem: período 1983 a 1996. Rev. LatinoAmerican. Enferm. 1998 Jan; 6 (1): 91-7.

14 Thiengo MA, Oliveira DC, Rodrigues BMRD. Adolescentes, AIDS e práticas de proteção: uma abordagem estrutural das Representações Sociais. Rev. Enferm. UERJ. 2002 Maio-Ago; 10 (2): 81-4.

15 Stefanelli MC, Cadete MMM, Aranha MI. Proposta de ação educativa na prevenção da AIDS: jogo educativo. Texto Contexto Enferm. 1998 Set-Dez; 7 (3): 158-73. 